\title{
A meta-analysis of traditional and functional end-to-side anastomosis in radiocephalic fistula for dialysis access
}

\author{
Tang Weigang ${ }^{1,2} \cdot$ Xu Wei $^{1,2} \cdot$ Gong Lifeng ${ }^{1,2} \cdot$ Lu Jingkui $^{1,2} \cdot$ Li Yani $^{1,2} \cdot$ Jiang Huaqin $^{1,2} \cdot$ Li Hui $^{1,2}$
}

Received: 26 July 2020 / Accepted: 27 October 2020 / Published online: 2 January 2021

(c) The Author(s) 2021

\begin{abstract}
Objective Functional vein end to arterial side (ETS) anastomosis uses vein side to arterial side (STS) anastomosis with distal vein ligation, which can achieve similar effects as those of ETS after STS anastomosis. The purpose of the study was to provide a meta-analysis to compare the clinical outcomes between traditional and functional ETS anastomosis in radiocephalic fistula for dialysis access.

Methods Databases including PubMed, EMbase, the Cochrane Library, CNKI, Wanfang database were searched from the inception to February 6, 2020. Eligible studies comparing traditional and functional ETS anastomosis in radiocephalic fistula were included. Data were analyzed using Review Manager Version 5.3.

Results Seven studies were included in the meta-analysis. Five randomized controlled trials and two cohort studies involving 841 patients were identified. Compared with traditional ETS anastomosis, functional ETS anastomosis had shorter anastomosis time (MD $-9.54,95 \% \mathrm{CI}-17.96$ to $-1.12, P=0.03$ ), higher surgical success rate (OR 3.80, 95\% CI 1.76-8.22, $P<0.01$ ), fewer complications(OR $0.18,95 \%$ CI $0.08-0.39, P<0.01$ ), higher patency rate after 3 months (OR $4.91,95 \%$ CI 1.19-20.33, $P=0.03$ ), higher patency rate after 6 months (OR 1.90, 95\% CI 1.09-3.31, $P=0.02$ ), higher patency rate after 12 months (OR 1.70, 95\% CI 1.09-2.66, $P=0.02$ ). There was no difference after the two arteriovenous (AVF) anastomosisl methods concerning AVF maturation time (SMD - 0.48, 95\% CI - 1.30-0.34, $P=0.25$ ) and patency rate after 1 month (OR 1.77, 95\% CI 0.65-4.80, $P=0.26$ ).

Conclusion Functional ETS anastomosis had advantages of easy operation, high surgical success rate, few complications, high patency rate of 3 months and long-term, but did not have obvious advantage in the early stages concerning AVF maturation time and 1-month patency rate.
\end{abstract}

Keywords Arteriovenous fistula $\cdot$ Functional end-to-side anastomosis $\cdot$ Dialysis access $\cdot$ Meta-analysis

\section{Introduction}

Autogenous arteriovenous fistula (AVF) is the preferred method of choice to achieve vascular access for long-term hemodialysis, because it has a low rate of complications and increases overall survival compared to other types of vascular access [1-7]. The radiocephalic fistula is considered

Xu Wei

q844055361@qq.com

1 Department of Nephrology, Wujin Hospital Affiliated with Jiangsu University, Changzhou City, Jiangsu Province, China

2 Department of Nephrology, The Wujin Clinical College of Xuzhou Medical University, Changzhou City, Jiangsu Province, China first in the establishment of arteriovenous fistula [8]. The common types of AVF anastomosis used in uremic patients are vein end to arterial end (ETE), vein side to arterial end (STE), vein end to arterial side (ETS), and vein side to arterial side (STS) [9]. In clinical practice, ETS anastomosis is a most common type because of higher proximal venous flow, longer fistula survival and lesser long-term complications $[10,11]$. European Society for Vascular Surgery (ESVS) guidelines also recommend ETS anastomosis [12].

In recent years, some scholars reported a modified AVF anastomosis, which had a good result. This modified AVF anastomosis was named functional ETS anastomosis using side-to-side anastomosis with distal vein ligation, which achieved similar effects as those of ETS after STS anastomosis. Our meta-analysis was conducted to compare the 
clinical outcomes between traditional and functional ETS anastomosis in radiocephalic fistula for dialysis access.

\section{Materials and methods}

\section{Search strategy}

Our meta-analysis has been reported in line with PRISMA (Preferred Reporting Items for Systematic Reviews and Meta-Analyses) and AMSTAR (Assessing the methodological quality of systematic reviews) Guidelines. Our meta-analysis was registered at the International Prospective Register of Systematic Reviews (Registration number: CRD42020166331).

We searched PubMed, Embase, the Cochrane Library, CNKI (China National Knowledge Infrastructure) and Wanfang from the inception to February 6, 2020. The combined text and MeSH terms included Arteriovenous Fistula, AVF, Access, Hemodialysis, End to Side, Functional, Modified. In addition, the cited papers and relevant references were searched manually to identify eligible studies. There were no language restrictions.

\section{Inclusion and exclusion criteria}

The inclusion criteria: (1) Randomized controlled trials (RCTs), cohort or case-control studies; (2) Hemodialysis patients; (3) Studies that compared traditional and functional ETS anastomosis in radiocephalic fistulas; Functional ETS anastomosis using side-to-side anastomosis with distal vein ligation (Fig. 1); (4) The main endpoint of the review was AFV patency rates. Secondary endpoints were anastomosis time, successful rate of surgery, maturation time, complications.

The exclusion criteria: (1) Severe cardiac insufficiency or poor conditions of vascular; (2) Case series, comments, reviews; (3) Lack of relevant outcomes data.

\section{Data extraction and quality assessment}

Data were extracted independently by two investigators using standard data extraction forms. In the case of disagreement, a third investigator was consulted. We extracted characteristics including first author, year of publication, location, study design, follow-up period, age, sex, sample size, anastomosis time, successful rate of surgery (The primary fistula patency was defined as the immediate success rate of surgery), maturation time of AVF (AVF maturation was defined as venous diameter $>0.6 \mathrm{~cm}$ and access flow $>600 \mathrm{~mL} / \mathrm{min}$ [1]), complications (Hemorrhage, infection, hand swelling, thrombosis, venous stenosis), patency rate (The primary patency rate was defined as the unassisted patency rate without endovascular and open surgery; The secondary patency rate was defined as the assisted patency rate with salvage procedures). The Cochrane assessment tool was used to evaluate the quality of RCTs [13]. The Newcastle-Ottawa scale (NOS) was used to evaluate the quality of non-randomized studies [14].
Fig. 1 a Traditional ETS anastomosis uses vein end to arterial side anastomosis. b Functional ETS anastomosis uses side-toside anastomosis with distal vein ligation. a cephalic vein; $b$ radial artery

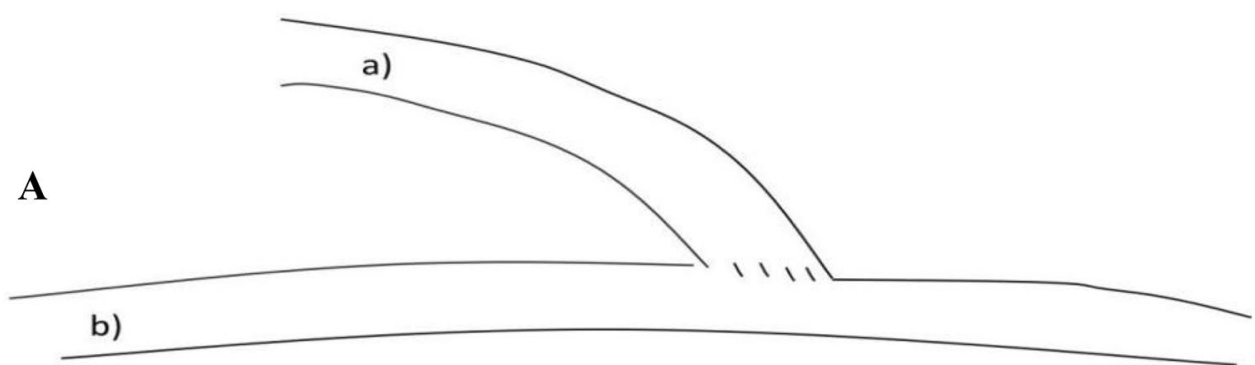

B

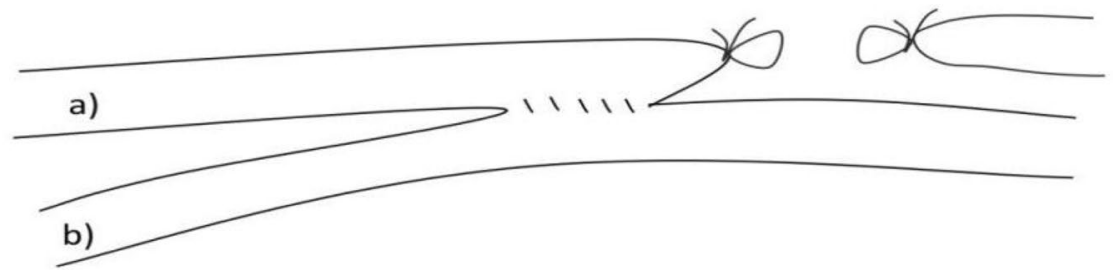




\section{Statistical analysis}

We performed the data analysis using Review Manager Version 5.3 (Cochrane Collaboration). Heterogeneity between studies was assessed by using $I^{2}$ statistics. We considered $I^{2}>50 \%$ and $P<0.10$ to imply significant heterogeneity [15]. Homogeneous data was performed using the fixedeffects model. Heterogeneous data was performed using the random-effects model. We presented categorical variables as Odds Ratios (OR). Continuous data were presented as the mean difference (MD). Summary estimates and $95 \%$ confidence intervals (CIs) were calculated. Overall effects were determined by the using $Z$-test. A $P$ value $<0.05$ was considered significant. Publication bias was assessed using sensitivity analysis.

\section{Results}

\section{Study selection and characteristics}

A flow diagram of the selection process is shown in Fig. 2. Finally, six studies from China [16-21] and one study from America [22] were included in this analysis. Of the seven studies, five were RCTs and two were cohort studies. Five studies were published in the Chinese journal. As a whole,
458 patients were included in the functional ETS anastomosis group and 383 patients were included in the traditional ETS anastomosis group. The follow-up period was from 6 months to 2 years. The risk of bias in included RCTs was moderate. The cohort studies achieved scores of $\geq 6$ points, which were considered to be of high quality. The baseline characteristics of these studies are listed in Tables 1 . The NOS assessment is listed in Table 2 and the Cochrane assessment is listed in Table 3.

\section{Meta-analysis results}

Anastomosis time Data about anastomosis time were reported in four articles [16-18, 21]. There was significant heterogeneity among the studies $\left(P<0.01, I^{2}=97 \%\right)$, so finally the random-effects model was used for the metaanalysis. Anastomosis time of the functional ETS group was shorter than the traditional STE group (MD - 9.54, 95\% CI -17.96 to $-1.12, P=0.03$ ) (Fig. 3).

Succesful rate of surgery Data about successful rate of surgery were reported in six articles [16-21], 417/426 (97.9\%) for the functional ETS group and 328/417 (92.7\%) for the traditional ETS group. The heterogeneity among these studies was not substantial $\left(P=0.74, I^{2}=0 \%\right)$, so the fixedeffects model was used for the meta-analysis. Succesful
Fig. 2 Flow diagram of the literature search

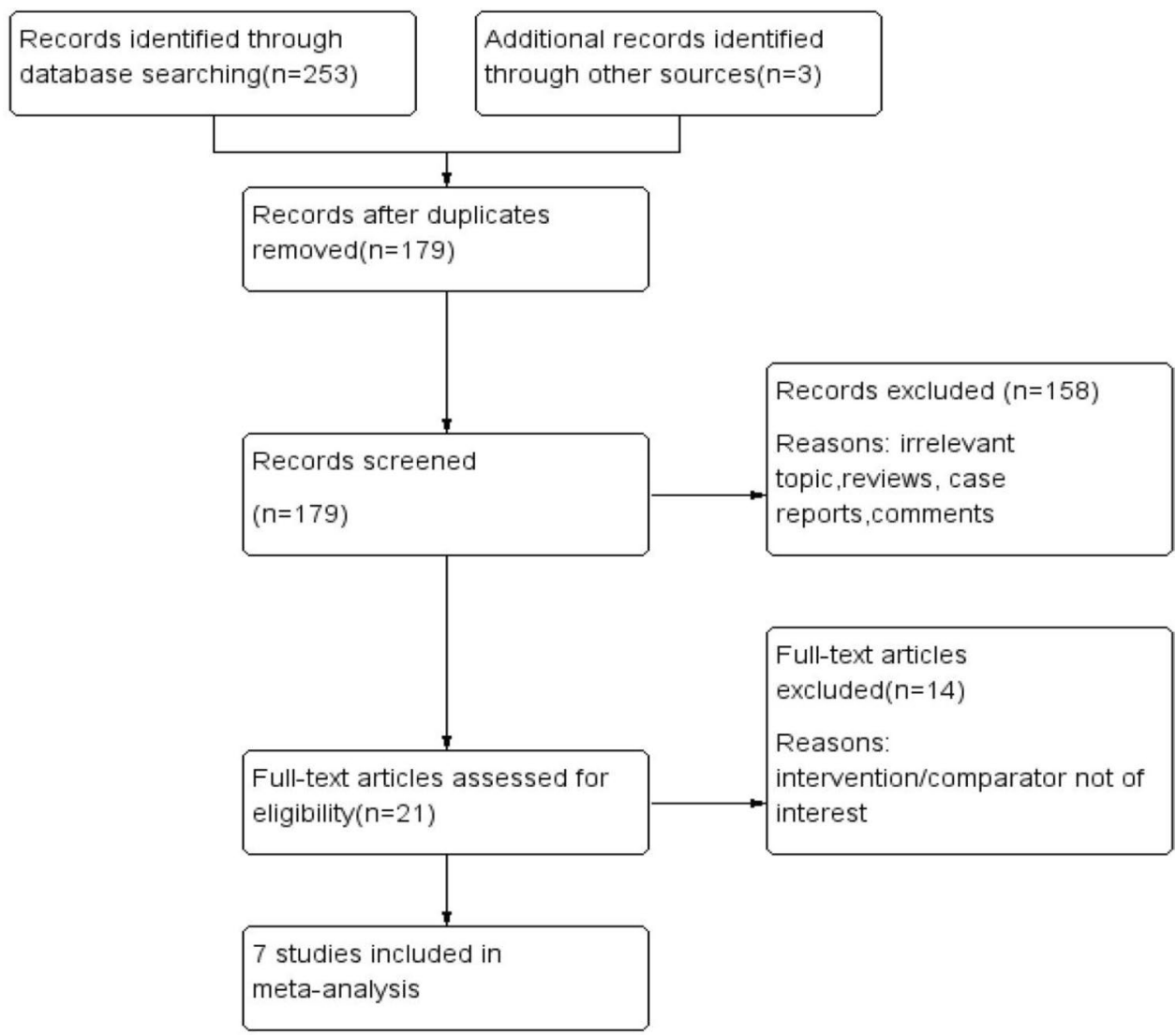




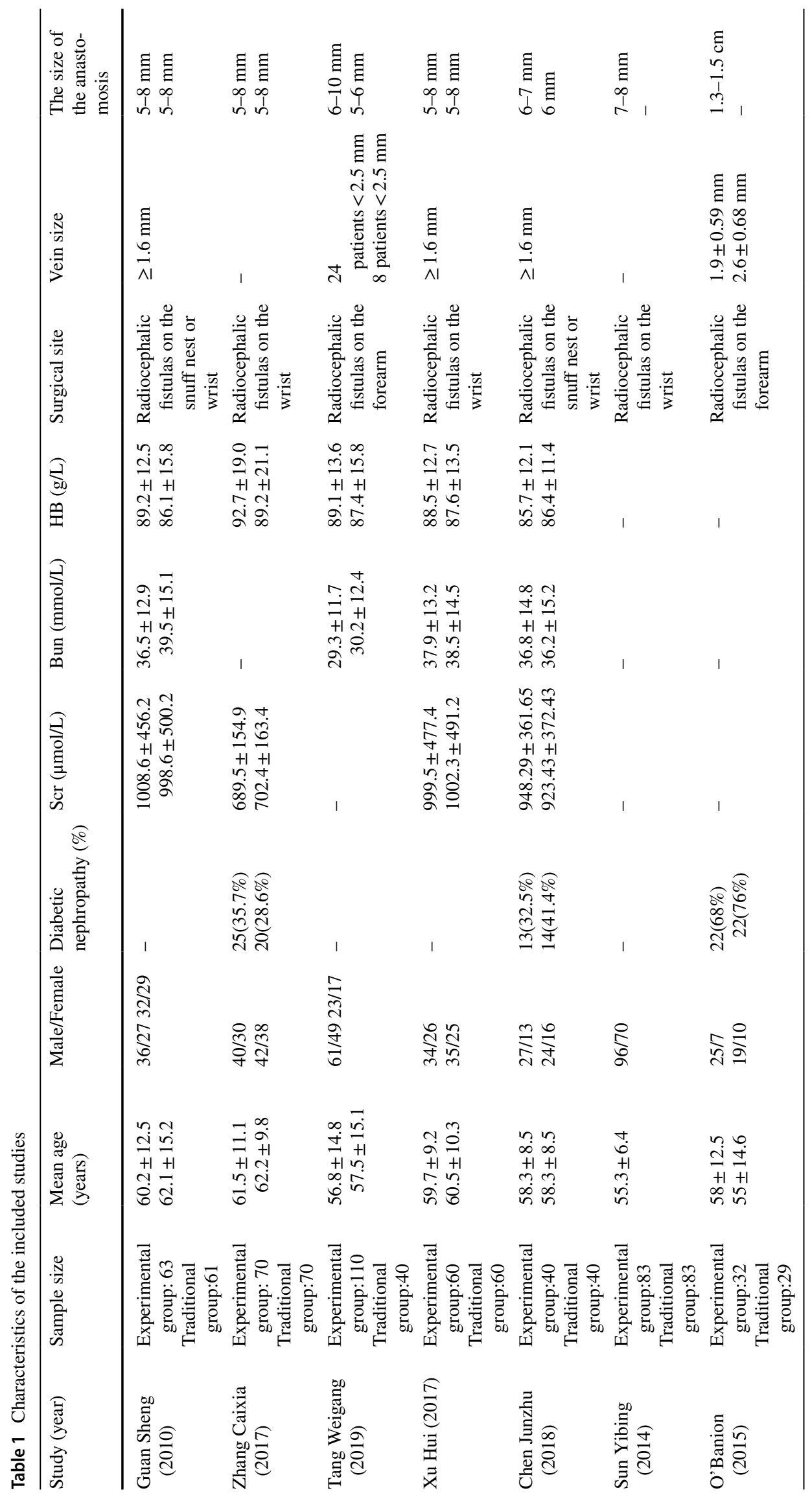


Table 2 Quality assessment of cohort studies

\begin{tabular}{lllll}
\hline Studies & Selection & Comparability & Outcome & Score \\
\hline Tang Weigang 2019 & $\star \star \star$ & $\star$ & $\star \star \star$ & 7 \\
O'Banion 2015 & $\star \star \star$ & $\star$ & $\star$ & 6 \\
\hline
\end{tabular}

rate of surgery in the functional STE group was higher than the traditional ETS group (OR 3.80, 95\% CI 1.76-8.22, $P<0.01$ ) (Fig. 4).

Maturation time of AVF Data about maturation time of AVF were reported in two articles $[17,18]$. There was significant

Table 3 Risk of bias of randomized control trial

\begin{tabular}{|c|c|c|c|c|c|c|}
\hline Study & $\begin{array}{l}\text { Random sequence } \\
\text { generation }\end{array}$ & $\begin{array}{l}\text { Allocation con- } \\
\text { cealment }\end{array}$ & $\begin{array}{l}\text { Blinding of participants } \\
\text { and personnel }\end{array}$ & $\begin{array}{l}\text { Incomplete out- } \\
\text { come data }\end{array}$ & Selective reporting & Other bias \\
\hline $\begin{array}{l}\text { Zhang Caixia } \\
(2017)\end{array}$ & Low risk & Unclear & Unclear & Low risk & Low risk & Unclear \\
\hline $\begin{array}{l}\text { Xu Hui } \\
(2017)\end{array}$ & Low risk & Low risk & Low risk & Low risk & Low risk & Unclear \\
\hline $\begin{array}{l}\text { Guan Sheng } \\
\text { (2010) }\end{array}$ & Low risk & Low risk & Low risk & Low risk & Low risk & Unclear \\
\hline $\begin{array}{l}\text { Chen Junzhu } \\
\text { (2018) }\end{array}$ & Low risk & Unclear & Unclear & Low risk & Low risk & Low risk \\
\hline $\begin{array}{l}\text { Sun Yibing } \\
(2014\end{array}$ & Unclear & Unclear & Unclear & Low risk & Low risk & Unclear \\
\hline
\end{tabular}

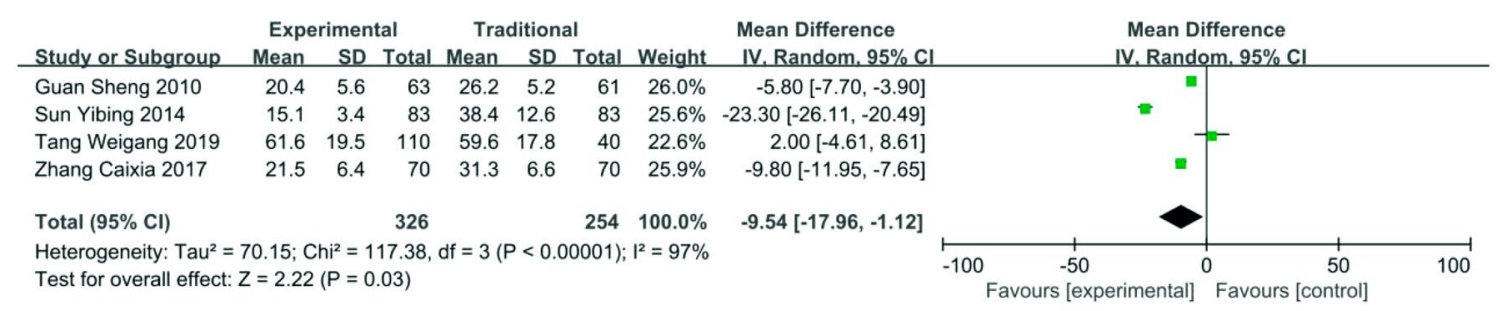

Fig. 3 Forest plot of anastomosis time between functional ETS group and traditional ETS group. Control group: traditional ETS anastomosis; Experimental group: functional ETS anastomosis

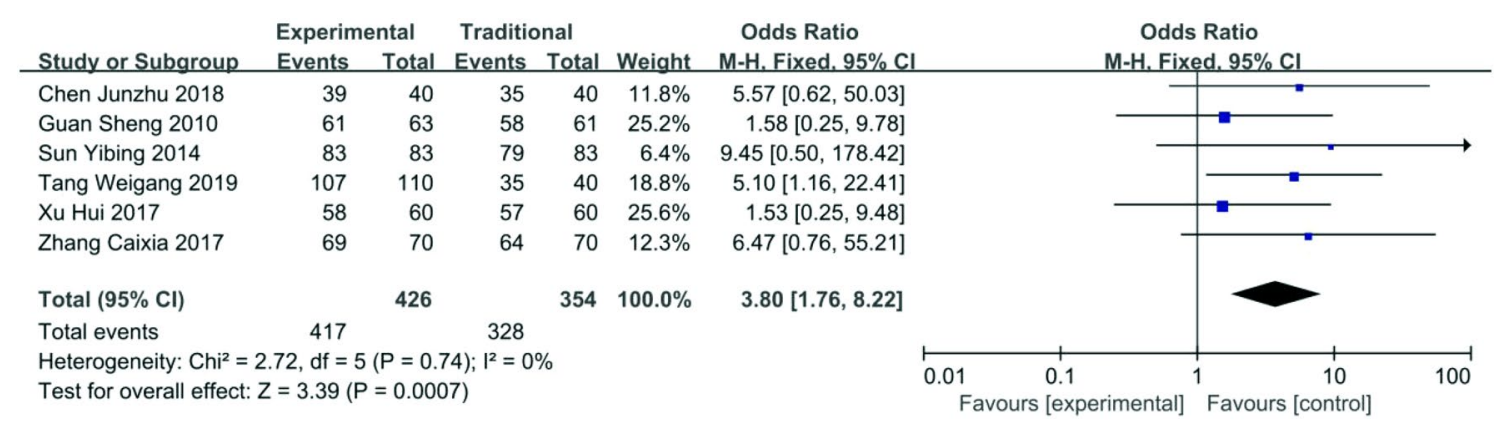

Fig. 4 Forest plot of succesful rate of surgery between functional ETS group and traditional ETS group. Control group: traditional ETS anastomosis; Experimental group: functional ETS anastomosis 
heterogeneity between the two studies $\left(P<0.01, I^{2}=90 \%\right)$, so finally the random-effects model was used for the metaanalysis. There was no significant difference between both groups concerning maturation time of AVF (SMD $-0.48,95 \%$ CI $-1.30-0.34, P=0.25$ ) (Fig. 5).

Patency rate after 1 month Data about patency rate after 1 month were reported in two articles [16, 18], 165/173 (95.4\%) for the functional ETS group and 93/101 (92.0\%) for the traditional STE group. The heterogeneity between the two studies was not substantial $\left(P=0.13, I^{2}=57 \%\right)$, so the fixed-effects model was used for the meta-analysis. There was no significant difference between both groups concerning patency rate after 1 month (OR 1.77, 95\% CI 0.65-4.80, $P=0.26$ ) (Fig. 6).

Patency rate after 3 months Data about patency rate after 3 months were reported in two articles [20, 22], 70/72 (97.2\%) for the functional ETS group and 59/69 (85.5\%) for the traditional ETS group. The heterogeneity between the two studies was not substantial $\left(P=0.47, I^{2}=0 \%\right)$, so the fixed-effects model was used for the meta-analysis. Patency rate after 3 months in the functional ETS group was higher than the traditional ETS group (OR 4.91, 95\% CI 1.19-20.33, $P=0.03$ ) (Fig. 7).

Patency rate after 6 months Data about patency rate after 6 months were reported in four articles [16, 19, 20, 22], $170 / 195(87.1 \%)$ for the functional STE group and 150/190 (78.9\%) for the traditional ETS group.The heterogeneity among these studies was not substantial $\left(P=0.29, I^{2}=20 \%\right)$, so the fixed-effects model was used for the meta-analysis. Patency rate after 6 months in the functional ETS group was higher than the traditional ETS group (OR 1.90, 95\% CI 1.09-3.31, $P=0.02$ ) (Fig. 8).

Patency rate after 12 months Data about patency rate after 12 months were reported in five articles [16-20], 301/343 (87.7\%) for the functional ETS group and 213/271 (78.6\%) for the traditional ETS group. The heterogeneity among these studies was not substantial $\left(P=0.27, I^{2}=23 \%\right)$, so the fixed-effects model was used for the meta-analysis. Patency

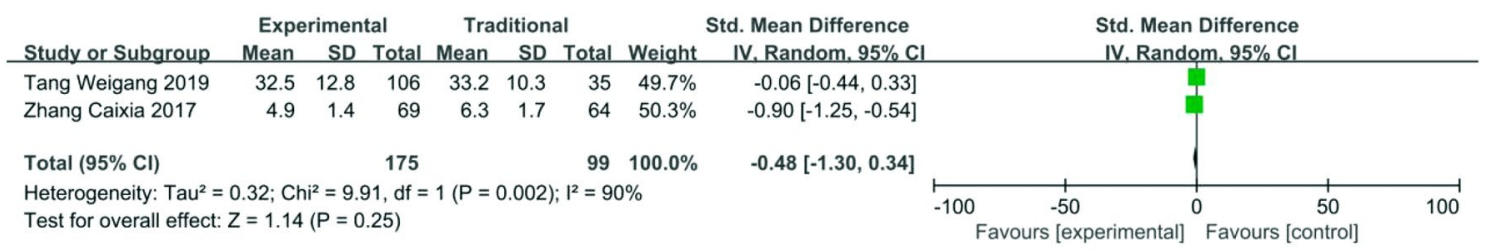

Fig. 5 Forest plot of maturation time of AVF between functional ETS group and traditional ETS group. Control group: traditional ETS anastomosis; Experimental group: functional ETS anastomosis

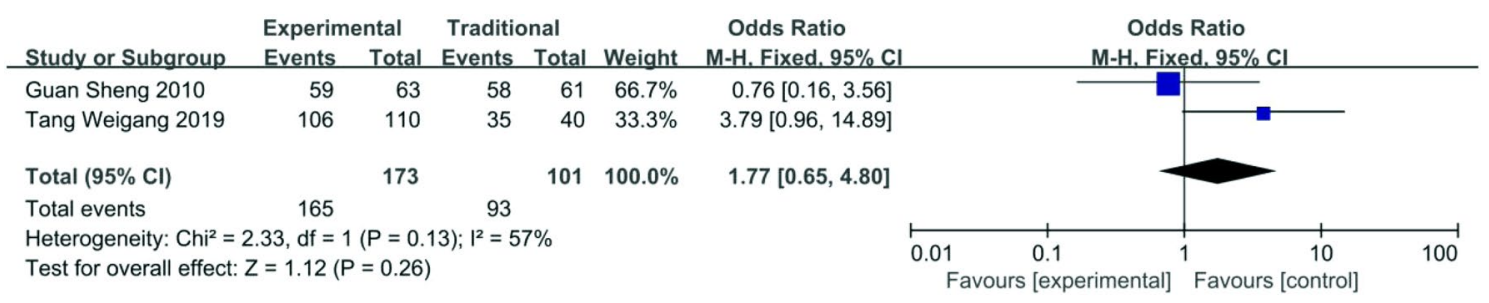

Fig. 6 Forest plot of patency rate after 1 month between functional ETS group and traditional ETS group. Control group: traditional ETS anastomosis; Experimental group: functional ETS anastomosis

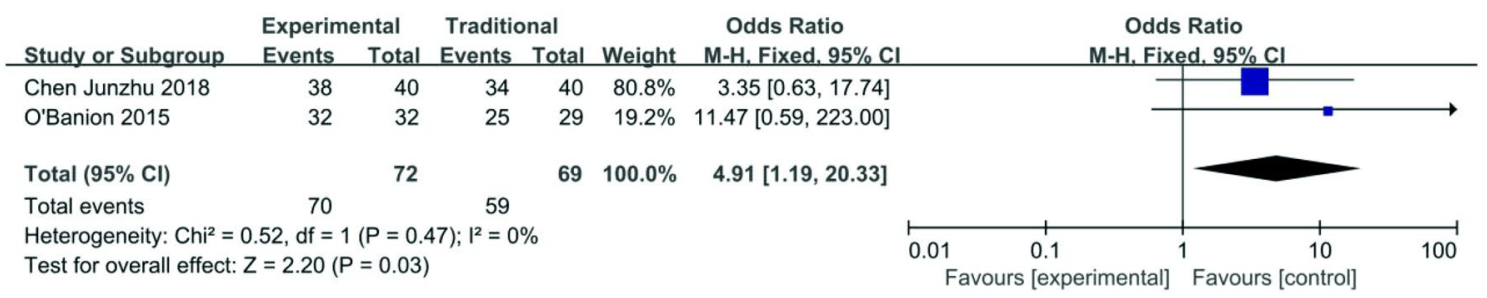

Fig. 7 Forest plot of patency rate after 3 month between functional ETS group and traditional ETS group. Control group: traditional ETS anastomosis; Experimental group: functional ETS anastomosis 
rate after 12 months in the functional ETS group was higher than the traditional ETS group (OR 1.70, 95\% CI 1.09-2.66, $P=0.02$ ) (Fig. 9).

Complications Data about complications were reported in four articles [16, 19-21]. Incidences of hemorrhage $(1.40 \%, 2 / 143)$, infection $(1.40 \%, 2 / 143)$, hand swelling $(0.55 \%, 1 / 183)$, early thrombosis $(1.00 \%, 1 / 101)$,thrombosis $(2.11 \%, 3 / 142)$, venous stenosis $(0 \%, 0 / 110)$ in the functional ETS group were all lower than incidences of hemorrhage $(6.99 \%, 10 / 143)$, infection $(6.29 \%, 9 / 143)$, hand swelling $(1.09 \%, 2 / 183)$,early thrombosis $(10.10 \%, 10 / 99)$, thrombosis $(11.27 \%, 16 / 142)$, venous stenosis $(3.96 \%, 4 / 101)$ in the traditional ETS group. The total incidence of complications were 8/246 (3.3\%) for the functional ETS group and 39/244 (15.9\%) for the traditional ETS group. The heterogeneity among these studies was not substantial $\left(P=0.95, I^{2}=0 \%\right)$, so the fixed-effects model was used for the meta-analysis. Complications of the functional ETS group were fewer than the traditional ETS group (OR 0.18, 95\% CI 0.08-0.39, $P<0.01$ ) (Fig. 10).

\section{Publication bias}

For heterogeneous studies, the number of included studies (less than 3) was too small to conduct any sufficient analysis

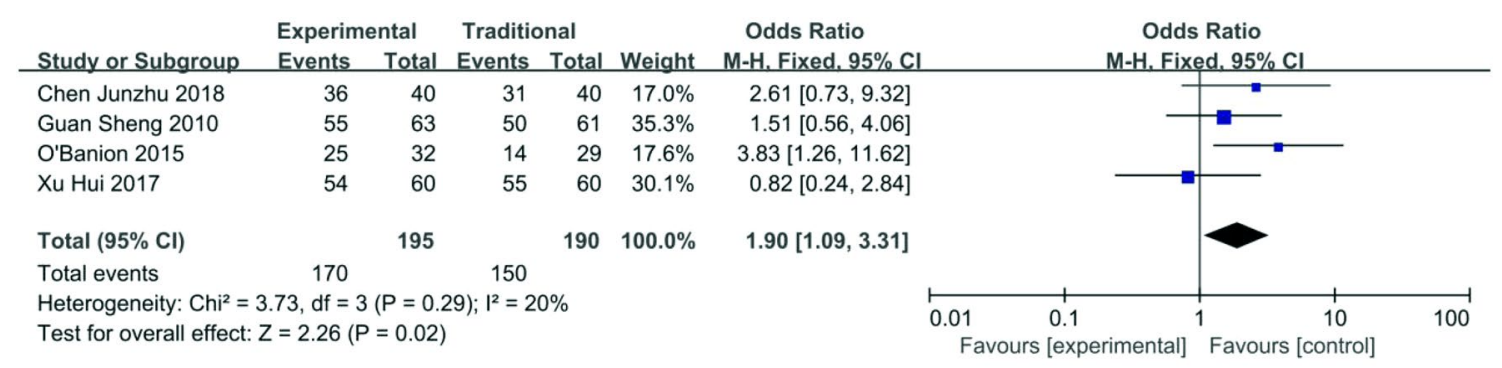

Fig. 8 Forest plot of patency rate after 6 month between functional ETS group and traditional ETS group. Control group: traditional ETS anastomosis; Experimental group: functional ETS anastomosis

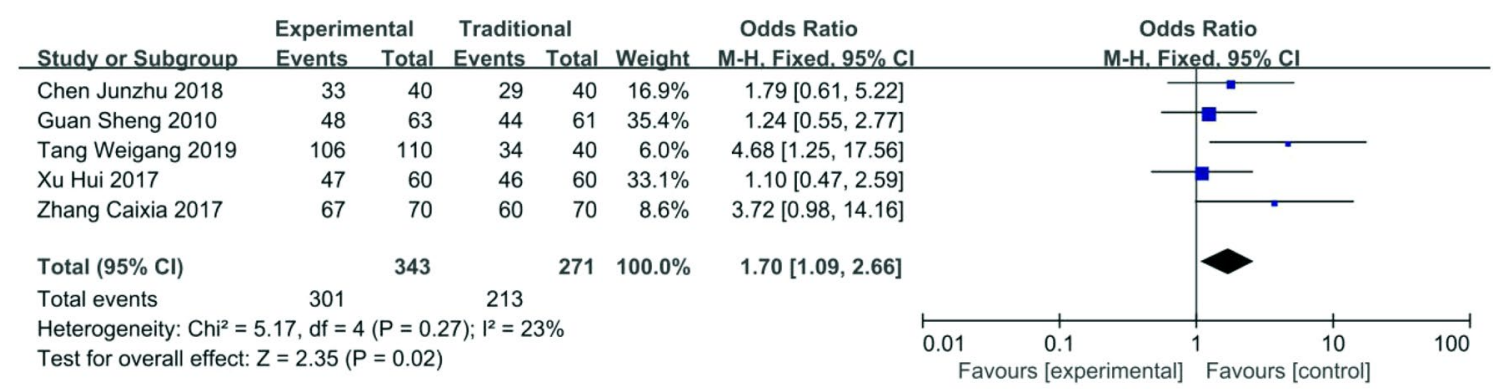

Fig. 9 Forest plot of patency rate after 12 months between functional ETS group and traditional ETS group. Control group: traditional ETS anastomosis; Experimental group: functional ETS anastomosis

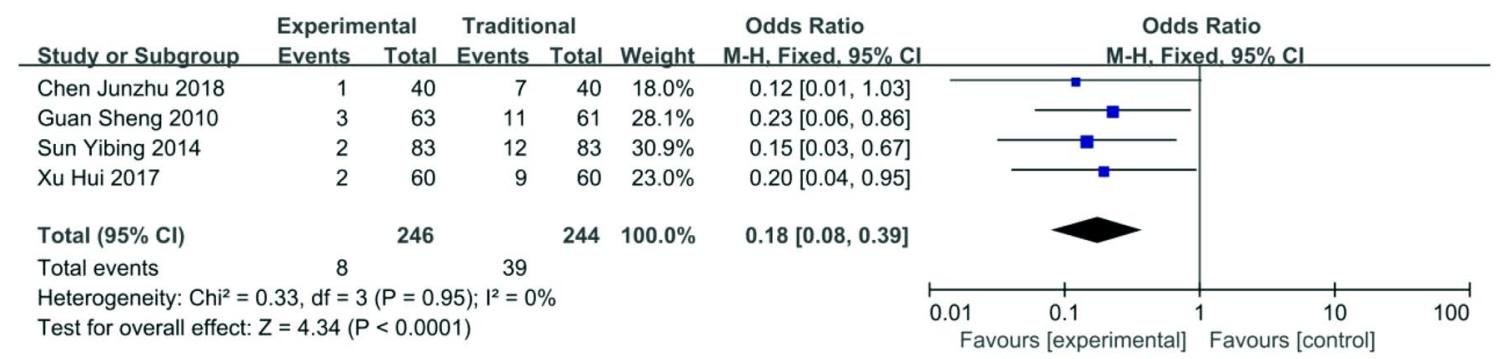

Fig. 10 Forest plot of complications between functional ETS group and traditional ETS group. Control group: traditional ETS anastomosis; Experimental group: functional ETS anastomosis 
of publication bias. Remarkably, The majority of participants in O'Banion study were not Asian, which was different from other studies. If we deleted O'Banion study, patency rates after 3 months $(P=0.15)$ and 6 months $(P=0.23)$ were no difference between the two groups.

\section{Discussion}

The well-functioning AVF provides enough blood flow without complications for a long time, which is the key to successful hemodialysis. Considering the limitation of vascular resources, the radiocephalic fistula is the most common choice of initial vascular access. The most commonly reported techniques of AVF anastomosis are ETE, STE, ETS and STS.ETE and STE anastomosis has the low fistula flow rate [23], which are used less in clinical practice.STE anastomosis also has the greatest risk of venous hypertension [24]. ETS anastomosis is a highly recommended anastomosis type because it has a high fistula flow and the low risk of venous hypertension of the hand [25]. STS anastomosis is the easiest construct technique and used commonly, which has the highest fistula flow [24]. However, STS anastomosis has the highest risk of venous hypertension. The most effective solution of venous hypertension is ligation of the distal venous arm [24]. The procedure of functional ETS anastomosis is STS anastomosis followed by distal cephalic vein ligation or distal cephalic vein ligation followed by STS anastomosis. It has a similar effect of traditional ETS anastomosis and has advantages of STS anastomosis at the same time. Sung and Zafar-Ul reported that one-year patency rates of AVF using functional STE anastomosis were both nearly $90 \%$ [26, 27]. Sun Yibing reported that two years patency rate of AVF using functional ETS anastomosis achieved 90\% [21]. Our meta-analysis revealed that there was no difference between the two AVF anastomosis methods concerning maturation time and 1-month patency rate. Compared with the traditional ETS anastomosis, the advantages of the functional ETS anastomosis were shorter anastomosis time, higher surgical success rate, 3-12 months patency rate.

The recognized factors affecting the maturation and patency of AVF are reported to venous blood flow and the size of the vein $[28,29]$. Sufficient venous blood flow and vein sizes will lead to reduce the risk of thrombosis, promote early maturation and increase the patency rate. If the fistula does not develop early thrombosis within three months, the risk of overall thrombosis is decreasing drastically [30]. Creating an fistula with early maturation and patency can reduce usage time of hemodialysis catheter, hospital stays and overall cost. In the procedure of functional ETS anastomosis, using STS anastomosis can reduce the impact of vein size and create large anastomotic diameter, which allows for larger venous blood flow. In the studies of Tang Weigang and O'Banion, the vein size in functional ETS anastomosis group were obviously smaller than in traditional ETS anastomosis group, but anastomotic diameter in functional ETS anastomosis group were obviously larger than in traditional ETS anastomosis group [18, 22]. In the traditional ETS anastomosis, some veins of small inner diameter cannot achieve ideal anastomotic caliber even after mechanical expansion and bevel pruning. The traditional ETS anastomosis is limited by the diameter of the vein. This explains that functional ETS anastomosis had higher 3 month patency rate and simultaneously long-term (6-12 months) patency rate. In our meta-analysis, average maturation time in functional ETS anastomosis was shorter than in traditional ETS anastomosis and 1-month patency rate in functional ETS anastomosis was higher than in traditional ETS anastomosis. However, there was no statistical difference concerning maturation time and 1-month patency. For the analysis about maturation time and 1-month patency rate, the number of included studies were only two, which might not be adequate to judge conclusion.

Many operators of functional ETS anastomosis summarize the experience of operation in their studies. First, the operator of functional ETS anastomosis need not turnover vessel repeatedly because the position of arteries and veins is relatively stationary and paralleled, which can reduce arteriovenous angulation and reduce risk of poor arteriovenous alignment, vascular distortion and rotation. Second, the operator of functional ETS anastomosis need not pruner anastomotic surface of vein overmuch, which can save part of the operation time and reduce the damage to the vessel [17-21]. These two points can also explain that the functional ETS anastomosis reduces the risk of thrombosis, has higher surgical success rate and improve the patency rate. Furthermore, in the case of postoperative AVF thrombosis, we can open the ligation line of the distal vein to explore and remove thrombosis.

There were some limitations in our meta-analysis. First, patency rates in most of the included studies were not defined clearly as primary or secondary patency rate. One year AVF patency rate of functional ETS anastomosis in our meta-analysis were $87.7 \%$. The Tang's study performed in our hospital was a clue that one-year primary patency rate of the functional ETS anastomosis was $96.4 \%$ (106/110) [18]. Second, gender, age, diabetes mellitus are also reported to be the risk factors affecting the function of AVF. These risk factors were not different between the two anastomosis methods in most studies. However, these risk factors were not counted in the few studies we do not know if there's a difference. Third, for the analysis about maturation time and 1-3 months patency rate, included studies had a small sample size, so our metaanalysis may not be adequate to judge the accuracy. Fourth, in the O'Banion study, the majority of participants were not Asian and patency rate after 6 months in the traditional ETS 
anastomosis group was only $48 \%$. If we deleted O'Banion study, 1-6 month patency rates were all no difference between the two groups. So publication bias was still possible.

\section{Conclusions}

Functional ETS anastomosis has a similar effect of traditional ETS anastomosis and has advantages of STS anastomosis at the same time. Our meta-analysis revealed that the advantages of functional ETS anastomosis were an easy operation, high surgical success rate, few complications, high patency rate of 3 months and long-term. AVF maturation time and 1-month patency rate of the two anastomosis methods might be similar. To further confirm the conclusion, more large multicenter randomized controlled trials comparing the two AVF anastomosis methods are necessary.

Funding There was no funding for this work.

\section{Compliance with ethical standards}

Conflict of interest The authors declare that they have no conflict of interest.

Open Access This article is licensed under a Creative Commons Attribution 4.0 International License, which permits use, sharing, adaptation, distribution and reproduction in any medium or format, as long as you give appropriate credit to the original author(s) and the source, provide a link to the Creative Commons licence, and indicate if changes were made. The images or other third party material in this article are included in the article's Creative Commons licence, unless indicated otherwise in a credit line to the material. If material is not included in the article's Creative Commons licence and your intended use is not permitted by statutory regulation or exceeds the permitted use, you will need to obtain permission directly from the copyright holder. To view a copy of this licence, visit http://creativecommons.org/licenses/by/4.0/.

\section{References}

1. National Kidney Foundation (2006) NKF-K/DOQI clinical practice guidelines for vascular access: update 2006. Am J Kidney Dis 48(suppl):S176-306

2. Vascular Access Work Group (2006) Clinical practice guidelines for vascular access. Am J Kidney Dis 148:S248-S273

3. Dixon BS, Novak L, Fangman J (2002) Hemodialysis vascular access survival: upper-arm native arteriovenous fistula. Am J Kidney Dis 39(1):92-101

4. Anel RL, Yevzlin AS, Ivanovich P (2003) Vascular access and patient outcomes in hemodialysis: questions answered in recent literature. Artif Organs 27(3):237-241

5. Tordoir J, Canaud B, Haage P et al (2007) EBPG on vascular access. Nephrol Dialysis Transpl 22:88-117

6. Bashar K, Healy DA, Elsheikh S et al (2015) One-stage vs two-stage brachio-basilic arteriovenous fistula for dialysis access: a systematic review and a meta-analysis. PLoS ONE 10(3):e0120154
7. Almasri J, Alsawas M, Mainou M et al (2016) Outcomes of vascular access for hemodialysis: a systematic review and metaanalysis. J Vasc Surg 64:236-243

8. Jindal K, Chan CT, Deziel C et al (2006) Hemodialysis clinical practice guidelines for the Canadian Society of Nephrology. J Am Soc Nephrol 17:S1-27

9. Rodríguez Hernández JA, González Parra E, Julián Gutiérrez JM et al (2005) Vascular access guidelines for hemodialysis. Nefrologia 25(Suppl 1):3-97

10. Wedgwood KR, Wiggins PA, Guillou PJ (1984) A prospective study of end-to-side vs side-to-side arteriovenous fistulas for haemodialysis. Br J Surg 71(8):640-642

11. Galic G, Kvesic M, Tomic Z et al (2008) The advantages of end-to-side arteriovenous anastomosis over the other two types of arteriovenous anastomosis in dialysis patients. Coll Antropol 32(1):109-114

12. Schmidli J, Widmer MK, Basile C et al (2018) Editor's choicevascular access: 2018 clinical practice guidelines of the European Society for Vascular Surgery (ESVS). Eur J Vasc Endovasc Surg 55:757-818

13. Furlan AD, Malmivaara A, Chou R et al (2015) Updated method guideline for systematic reviews in the Cochrane back and neck group. Spine 40:1660-1673

14. Stang A (2010) Critical evaluation of the Newcastle-Ottawa scale for the assessment of the quality of nonrandomized studies in meta-analyses. Eur J Epidemiol 25:603-605

15. Ioannidis JP (2008) Interpretation of tests of heterogeneity and bias in meta-analysis. J Eval Clin Pract 14:951-957

16. Guan S, Bao W, Duan Y et al (2010) Funcfional and traditional end-to-side anastomosis for arteriovenous fistula plasty in hemodialysis patients:a randomized controlled study. Acad J Second Military Med Univ 31(10):1065-1067

17. Zhang CX (2017) Application of functional end-to-side anastomosis for arteriovenous fistula angioplasty used in hemodialysis. Chinese J Integr Traditional Western Nephrol 18(9):808-809

18. Tang WG, Gong LF, Lu JK (2019) The clinical application of functional end-to-side anastomosis in the primary arteriovenous fistula. Chinese J Integr Traditional Western Nephrol 20(8):730-731

19. Xu H, Deng BH, Wang XM et al (2017) The difrerenees between funcfional end-to-side anastomosis and traditional end-to-side anastomosis in the establishment of arteriovenous fistula surgerg. Int Med Health Guidance News 23(8):1200-1202

20. Chen JZ, Feng XG (2018) The application of improved end-toside anastomosis and traditional end-to-side anastomosis in arteriovenous fistula. Clinical J Chinese Med 10(21):38-41

21. Sun YB, Feng J, Yang W (2014) Application of functional endto-side anastomosis in arteriovenous fistula for hemodialysis. $\mathrm{J}$ Ningxia Med Univ 36(9):1023-1025

22. O'Banion LA, Van Buren D (2015) Radiocephalic fistulas for hemodialysis: a comparison of techniques. Am Surg 81:341-344

23. Johnson G, Dart CH, Peters RM (1966) The importance of venous circulation in arteriovenous fistula. Surg Gynecol Obstet 123:995-1000

24. Bennion RS (1994) Autogenous arteriovenous fistula. In: Strandness DE, Breda AV (eds) Vascular diseases: surgical and interventional therapy. Churchill Livingstone, New York, p 1048

25. Connall TP, Wilson SE. Angioaccess, vascular access for hemodialysis. Eugene S Jr, Vreda AV, eds. Vascular Diseases: Surgical and Interventional Therapy. New York: Churchill Livingstone Inc 1994:1236.

26. Hong SY, Yoon YC, Cho KH et al (2013) Clinical analysis of radiocephalic fistula using side-to-side anastomosis with distal cephalic vein ligation. Korean J Thoracic Cardiovascular Surg 46(6):439-443 
27. Ahsan ZU, Waheed A, Zaeem FA (2010) Arteriovenous fistulas constructed using side-to-side anastomosis with ligation and division of distal venous arm; a tertiary care hospital experience. $\mathrm{J}$ Vascular Access 11(1):26-30

28. Mousa AY, Dearing DD, AbuRahma AF (2013) Radiocephalic fistula: review and update. Ann Vase Surg 27:370-378

29. Jang JY, Hwang BK, Ha WS (1991) A clinical study for the factors influencing early patency of internal arteriovenous fistula in chronic renal failure patients. J Korean Surg Soc 41:635-640
30. Burkhart HM, Cikrit DF (1997) Arteriovenous fistula for hemodialysis. Semin Vase Surg 10:162-165

Publisher's Note Springer Nature remains neutral with regard to jurisdictional claims in published maps and institutional affiliations. 